\title{
The antifungal compound butenafine eliminates promastigote and amastigote forms of Leishmania (Leishmania) amazonensis and Leishmania (Viannia) braziliensis
}

\author{
Adriana Bezerra-Souza a , Eduardo S. Yamamoto a , Márcia D. Laurenti a , \\ Susan P. Ribeiro ${ }^{\mathrm{b}, \mathrm{c}}$, Luiz Felipe D. Passero ${ }^{\mathrm{d}, *}$ \\ a Laboratory of Pathology of Infectious Diseases (LIM-50), Medical School, University of São Paulo, Avenida Dr. Arnaldo 455, 01246903 Cerqueira César, SP, Brazil \\ b Case Western Reserve University, Pathology Department, Cleveland, USA \\ c Division of Clinical Immunology and Allergy, LIM60, University of Sao Paulo School of Medicine, Sao Paulo, Brazil \\ d São Vicente Unit, Paulista Coastal Campus, Universidade Estadual Paulista Júlio de Mesquita Filho (UNESP), Praça Infante Dom Henrique, s/n, 11330-900 São Vicente, SP, Brazil
}

\section{A R T I C L E I N F O}

\section{Article history:}

Received 24 May 2016

Received in revised form 9 August 2016

Accepted 17 August 2016

Available online 18 August 2016

\section{Keywords:}

Leishmania (Leishmania) amazonensis

Leishmania (Viannia) braziliensis

Butenafine

Antileishmanial agent

Drug repurposing

\begin{abstract}
A B S T R A C T
The production of ergosterol lipid, important for the Leishmania membrane homeostasis, involves different enzymes. This pathway can be blocked to azoles and allylamines drugs, such as Butenafine. The aim of the present work was to evaluate the anti-leishmanicidal activity of this drug in 2 major species of Leishmania responsible for causing the American tegumentar leishmaniasis (L. (L.) amazonensis and L. (V.) braziliensis). Butenafine eliminated promastigote forms of $L$. amazonensis and $L$. braziliensis with efficacy similar to miltefosine, a standard antileishmania drug. In addition, butenafine induced alterations in promastigote forms of L. amazonensis that resemble programmed cell death. Butenafine as well as miltefosine presented mild toxicity in peritoneal macrophages, however, butenafine was more effective to eliminate intracellular amastigotes of both $L$. amazonensis and $L$. braziliensis, and this effect was not associated with elevated levels of nitric oxide or hydrogen peroxide. Taken together, data presented herein suggests that butenafine can be considered as a prototype drug able to eliminate $L$. amazonensis and L. braziliensis, etiological agents of anergic diffuse and mucocutaneous leishmaniasis, respectively.
\end{abstract}

(c) 2016 Elsevier Ireland Ltd. All rights reserved.

\section{Introduction}

The protozoan belonging to the Leishmania genus (Kinetoplastida: Trypanosomatidae) causes cutaneous and visceral leishmaniasis in humans as well as wild and domestic animals. In the New World, at least 14 different species are responsible to cause leishmaniasis, being the visceral form caused by $L$. (L.) infantum and the cutaneous form by species belonging to the Viannia or Leishmania subgenera. The species $L$. (L.) amazonensis and $L$. (V.) braziliensis that cause cutaneous forms of leishmaniasis possess the highest medical-epidemiological importance, because they can cause severe forms of leishmaniasis, the anergic diffuse and mucocutaneous leishmaniasis, respectively $[18,28]$. Although a broad array of species can be responsible to cause leishmaniasis, affecting humans and animals, the treatment is limited to a few drugs, such as antimonial, amphotericin B and miltefosine [11,31].

The pentavalent antimonial is the first choice of treatment for leishmaniasis, but presents side effects including gastrointestinal intolerance and cardiotoxicity, frequently leading to the patient to abandon the

\footnotetext{
* Corresponding author.

E-mail address: felipepassero@clp.unesp.br (L.F.D. Passero).
}

therapy. In addition, in some geographic areas, such as Bihar, high degree of drug-resistant parasites has been constantly detected [24,25]. In cases of drug intolerance or parasite resistance to antimonials, amphotericin B is used as a second choice drug. The treatment with amphotericin B is effective leading to the clearance of the parasite in 99.5\% of the treated patients [7,33], however amphotericin B-resistant parasites have been observed $[1,25]$. In addition, amphotericin B interacts with the host cellular membrane causing adverse effects, including fever, rigors, hypertension/hypotension, hypoxia, renal and gastrointestinal toxicities, thus limiting its use. Liposomal formulations of amphotericin B are already available for use, but at high costs, limiting its use in low-income regions that are highly affected by leishmaniasis $[12,32]$. The leishmanicidal action of miltefosine was characterized in the early 1980s, and studies so far have demonstrated that this drug is effective against different species of Leishmania leading to apoptosis and/or interfering with lipid signaling. In spite of that, the main concerns of the therapy with miltefosine are related to the gastrointestinal toxicity, and its teratogenicity in animal models. In addition, the toxicity associated with kidney and liver is frequently observed in patients [10]. Considering the lack of effective drugs with low side effects, new alternatives for therapy are needed. 
The sterol biosynthesis pathway is shared by Leishmania parasites and fungi. The products of this pathway, such as ergosterol and other 24-methyl sterols, are required for maintenance of membrane homeostasis. Host cells do not produce this type of lipids making this biochemical route an interesting target for drug development against Leishmania. This pathway is very complex and involves different enzymes. Certain antifungal drugs as azoles were previously shown to block the $\mathrm{C} 14 \alpha$-demethylase enzyme, such as ketoconazole, eliminating promastigote and amastigote of Leishmania sp. as well as Trypanosoma cruzi, a protozoa phylogenetically related to Leishmania $[4,13,17]$. Other enzymatic targets of ergosterol pathway also have been referred as potential new leishmanicidal compounds, such as squalene epoxidase enzyme, that converts squalene to lanosterol, an important precursor of ergosterol; and allylamines drugs, able to impair the activity of ergosterol. The most studied allylamine drug so far is the terbinafine, that was proved to present activity against promastigote and amastigote forms of $L$. braziliensis, L. mexicana and L. amazonensis $[26,33]$. However, in vivo studies have shown that terbinafine did not present therapeutic efficacy in visceral and cutaneous experimental leishmaniasis $[27,29]$. These and others results were useful to show that squalene epoxidase enzyme can be a very attractive target to be blocked aiming at impairing the parasite viability. This is a field yet to be explored aiming at identifying new potential anti-leishmanicidal compounds like other squalene epoxidase-blocking antifungal drugs. The butenafine ( $N$-4-tert-butylbenzyl- $N$-methyl-1naphthalenemethylamine hydrochloride) is one of them and belongs to benzylamine class of drugs, with structure and mode of action similar to allylamines, that ultimately will block the biosynthesis of ergosterol [8].

Considering that Leishmania parasites produce ergosterol in a similar pathway as fungi does, this work aimed at investigating the effect of butenafine drug against promastigote and intracellular amastigote forms of $L$. amazonensis and L. braziliensis. So far, this is the first work that evaluates the leishmanicidal potential of butenafine.

\section{Material and methods}

\subsection{Drugs}

Butenafine hydrochloride (purity $\geq 98 \%$ ) and choline hexadecyl phosphate (miltefosine, purity $\geq 98 \%$ ) were purchased from Sigma-Aldrich. Butenafine were solubilized in dimethyl sulfoxide (DMSO), and miltefosine in sterile sodium chloride $0.9 \%$.

\subsection{Parasites}

L. (L.) amazonensis parasite (MHOM/BR/73/M2269) and L. (V.) braziliensis (MHOM/BR/1995/M15280) parasites were kindly provided by Prof. Dr. Fernando T. Silveira from the cryobank of "Leishmaniasis Laboratory Prof. Dr. Ralph Laison”, Department of Parasitology, Evandro Chagas Institute, Ministry of Health, Belém, Pará, Brazil. These parasites were identified using monoclonal antibodies and isoenzyme electrophoretic profiles at the Leishmaniasis Laboratory of the Evandro Chagas Institute (Belém, Pará state, Brazil). Parasites were grown in M199 medium, supplemented with $10 \%$ heat-inactivated fetal bovine serum, $10 \mu \mathrm{g} / \mathrm{mL}$ of gentamicin, $1000 \mathrm{U} / \mathrm{mL}$ of penicillin, supplemented with $10 \%$ heat-inactivated fetal calf serum (Gibco), $0.25 \%$ hemin (Sigma-Aldrich) and $2 \%$ sterile male human urine at $25{ }^{\circ} \mathrm{C}$. Promastigote forms in the stationary phase were used.

\subsection{Animals}

Six to eight weeks old female BALB/c mice were obtained from Medical School of São Paulo University. This study was carried out in strict accordance with the recommendations in the Guide for the Care and Use of Laboratory Animals of the Brazilian National Council of Animal
Experimentation (http://www.cobea.org.br). The protocol was approved by the Committee on the Ethics of Animal Experiments of the Institutional Animal Care and Use Committee at the Medical School of São Paulo University (CEP 357/15). For all experimental procedure mice were anaesthetized with thiopental, administrated by intraperitoneal route $(1 \mathrm{mg} / 200 \mu \mathrm{L})$.

\subsection{Evaluation of anti-promastigote effect of Butenafine}

Promastigote forms of $L$. (L.) amazonensis and $L$. (V.) braziliensis $\left(2 \times 10^{7}\right.$ promastigotes $\left./ \mathrm{mL}\right)$ were incubated in 96-well culture plate in M199 medium with butenafine in a range of 315.0 to $2.5 \mu \mathrm{M}$, the standard drug miltefosine in the range of 245.0 to $1.9 \mu \mathrm{M}$. Negative control was cultivated in medium and DMSO as vehicle solution (never exceeding $1 \% \mathrm{v} / \mathrm{v}$ ). The parasites were incubated for $48 \mathrm{~h}$ at $25^{\circ} \mathrm{C}$. Then, the plate was washed with $200 \mu \mathrm{L}$ of sodium chloride $0.9 \%(\mathrm{w} / \mathrm{v})$ three times with centrifugation at $3000 \mathrm{rpm}, 10 \mathrm{~min}$ at $4{ }^{\circ} \mathrm{C}$, followed by addition of MTT (3-(4,5-dimethylthiazol-2-yl)-2,5-diphenyltetrazolium bromide) $(5.0 \mathrm{mg} / \mathrm{mL})$. Four hours later, $50 \mu \mathrm{L}$ of $10 \%$ sodium dodecyl sulfate (SDS) was added to each well. The plates were further incubated for $18 \mathrm{~h}$ and read by ELISA reader at $595 \mathrm{~nm}$. Effective concentration $50 \%$ $\left(E_{50}\right)$ was estimated using Graph Pad Prism 5.0 software.

\subsection{Ultrastructural alterations induced by butenafine in L. (L.) amazonensis promastigotes}

Promastigote forms of $L$. (L.) amazonensis $\left(2 \times 10^{7}\right.$ promastigotes/ $\mathrm{mL}$ ) were incubated in 96-well culture plate in M199 medium with the $\mathrm{EC}_{50}$ of butenafine $(34.10 \mu \mathrm{M})$, or miltefosine $(77.32 \mu \mathrm{M})$ for $48 \mathrm{~h}$, at $25^{\circ} \mathrm{C}$. Negative control was cultivated with medium and vehicle solution DMSO (never exceeding $1 \% \mathrm{v} / \mathrm{v}$ ). The plate was centrifuged at $3000 \mathrm{rpm}, 4^{\circ} \mathrm{C}, 10 \mathrm{~min}$ and washed three times with $200 \mu \mathrm{L}$ of sodium chloride $0.9 \%$. Then the pellets were resuspended in glutaraldehyde $2 \%$ and incubated at $4{ }^{\circ} \mathrm{C}$, during $60 \mathrm{~min}$. Parasites were post-fixed in $1 \%$ osmium tetroxide, and these materials were stained and block staining in $1 \%$ aqueous uranyl acetate overnight, dehydrated using alcohol. Then, samples were embedded in a polyester resin, thin sectioned with a LKB ultratome, double-stained by uranyl acetate and lead citrate (Ladd Research Industries), and examined with a Jeol 1010 (Tokyo, Japan) transmission electron microscope (TEM).

\subsection{Peritoneal macrophages culture and cytotoxicity assay}

Approximately $2 \times 10^{5}$ peritoneal macrophages from BALB/c mice were cultured in RPMI medium supplemented with $10 \%$ of fetal bovine serum (Gibco), 2 mM L-glutamine (Sigma-Aldrich, USA), $10 \mathrm{mM}$ Hepes (Sigma-Aldrich), $1 \mathrm{mM}$ sodium piruvate, $1 \% \mathrm{vol} / \mathrm{vol}$ nonessential amino acid solution (Gibco), $10 \mu \mathrm{g} / \mathrm{mL}$ of gentamicin and $1000 \mathrm{U} / \mathrm{mL}$ of penicillin in the presence of butenafine ( 315.0 to $2.5 \mu \mathrm{M}$ ) or miltefosine ( 245.0 to $1.9 \mu \mathrm{M})$. As negative control, macrophages were cultivated in medium and DMSO as vehicle solution (never exceeding $1 \% \mathrm{v} / \mathrm{v}$ ). After $48 \mathrm{~h}$, cell viability was analyzed by MTT method. Cytotoxic concentration 50\% ( $\left.\mathrm{CC}_{50}\right)$ was estimated, and Graph Pad Prism 5.0 software was used for plotting and statistical analysis.

\subsection{Macrophage infection and treatments, nitric oxide and cytokine determination}

Peritoneal macrophages from BALB/c mice $\left(2 \times 10^{5}\right.$ macrophage $)$ were cultivated in round cover slips in 24 -well plate, followed by infection with $L$. (L.) amazonensis or $L$. $(V$.$) braziliensis promastigotes at a$ ratio of 10 parasites per 1 peritoneal macrophage. Plates were incubated at $5 \% \mathrm{CO}_{2}$ at $35^{\circ} \mathrm{C}$. After $24 \mathrm{~h}$ of the initial infection, butenafine $(25 ; 50$ or $100 \mu \mathrm{M}$ ) or $\mathrm{EC}_{50}$ of miltefosine (estimated in $60 \mu \mathrm{M}$ for L. amazonensis and $64 \mu \mathrm{M}$ for $L$. braziliensis) were added to the infected culture. After $48 \mathrm{~h}$ of incubation supernatants were collected and stored at $-80^{\circ} \mathrm{C}$ 
for nitric oxide and hydrogen peroxide quantifications, according to manufacturer's instructions (Life Technologies, USA). Round cover slips were dried at room temperature, fixed in methanol, and stained by Giemsa. At least 100 macrophages were quantified and the degree of infection was evaluated by the Infection Index (II), estimated according to Passero et al. [22], following the expression:

$\mathrm{II}=\%$ infected macrophage $\times$ internalized amastigote forms/ macrophage

\subsection{Statistical analysis}

The results were expressed as the mean \pm standard deviation of three independent experiments and the nonparametric Mann-Whitney $U$ test was used to compare results among groups. Differences were considered statistically significant at $5 \%$ significance level $(p<0.05)$. GraphPad Prism 5 (GraphPad Software, Inc., La Jolla, CA, USA) was used to plot and analyze the results.

\section{Results}

\subsection{Antipromastigote activity of butenafine}

Butenafine eliminated the promastigote forms of $L$. amazonensis and L. braziliensis (Fig. 1A) in a dose-dependent manner, eliminating 50\% of promastigote forms of both species with an estimated concentration $50 \%\left(\mathrm{EC}_{50}\right)$ of $34.10 \pm 3.76$ and $81.25 \pm 10.24 \mu \mathrm{M}$, respectively. In a similar way, the standard drug miltefosine also eliminated promastigote forms in a dose-dependent fashion, showing $\mathrm{EC}_{50} \mathrm{~S}$ of $77.32 \pm 6.78$ and $61.43 \pm 9.37 \mu \mathrm{M}$ for $L$. amazonensis and L. braziliensis, respectively (Fig. 1B).
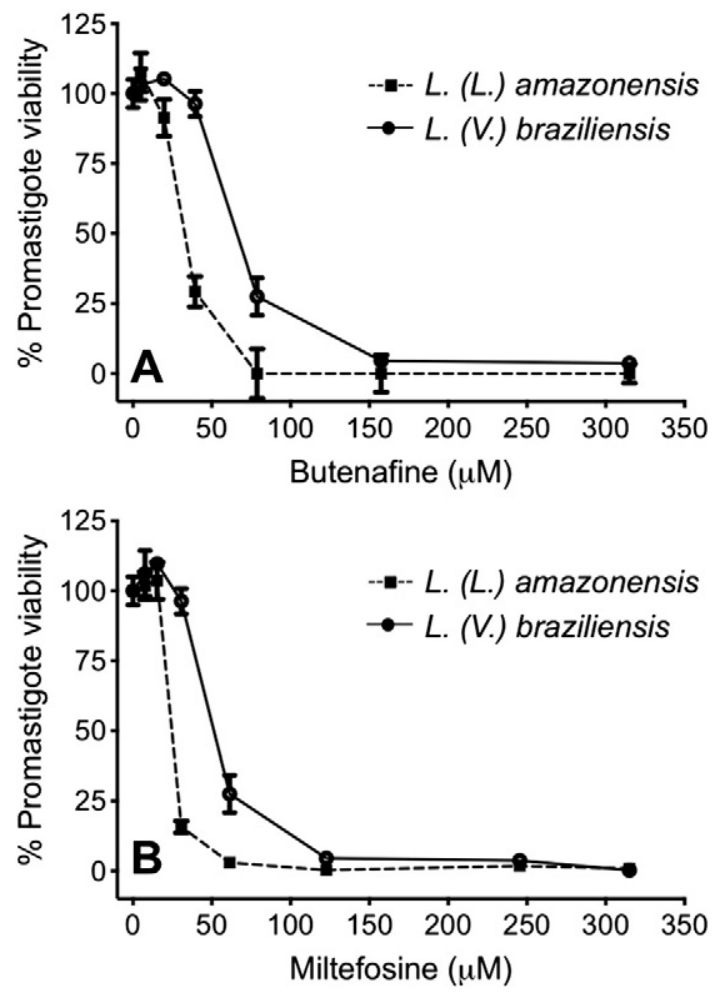

Fig. 1. Promastigote forms of $L$. amazonensis and $L$. braziliensis were incubated for $48 \mathrm{~h}$ with butenafine (A) or miltefosine (B) and the parasite viabilities were estimated by MTT method. Dashed lines $-L$. (L.) amazonensis; solid line $-L$. (V.) braziliensis.

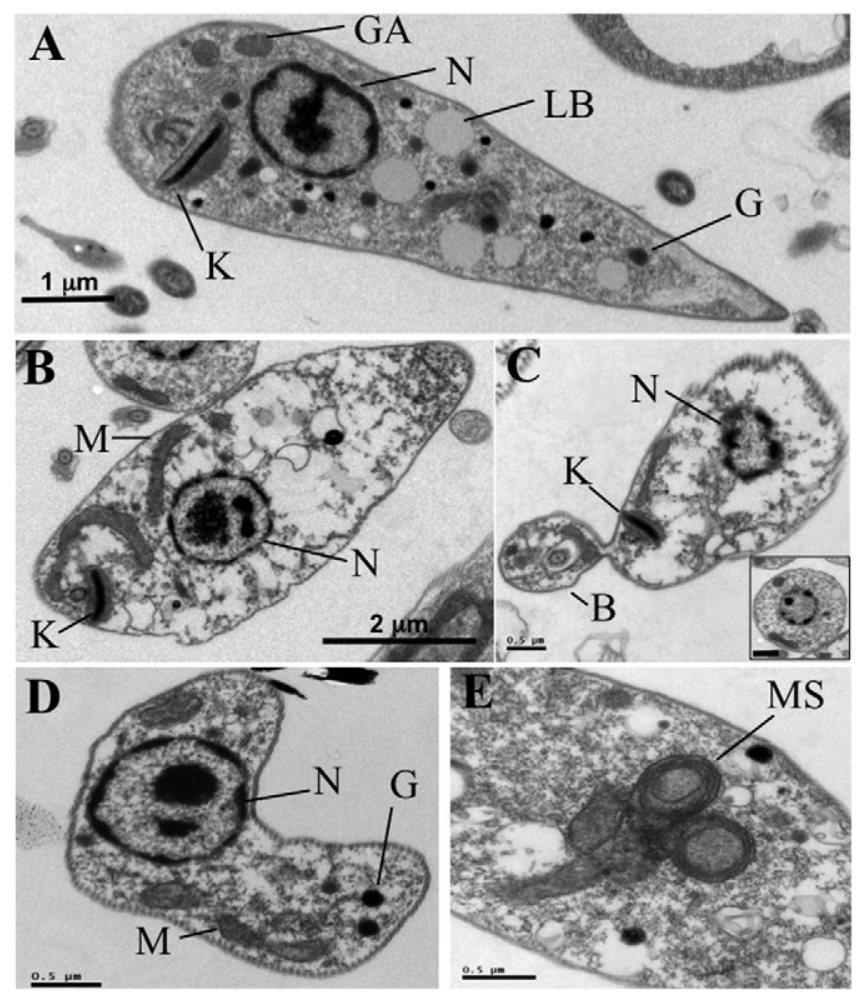

Fig. 2. Ultrastructural changes of $L$. (L.) amazonensis promastigote induced by treatment with Butenafine. Images of promastigote forms before (A) and after treatment with Butenafine $\mathrm{EC}_{50}(\mathrm{~B}$ and $\mathrm{C}$ ) or Miltefosine (D and $\mathrm{E}$ ) were captured by transmission electron microscopy. (N) - nucleus, (K) - kinetoplast, $\mathrm{M}$ - mitochondria, L - lipid body, G - glycosome, GA - Golgi apparatus, B - blebs, MS - myelin-like structure.

\subsection{Ultrastructural alterations induced by butenafine}

Control promastigotes (Fig. 2A) presented typical ultrastructural characteristics with a fusiform shape with well-preserved cell membrane, the cytoplasm presented a normal morphology, containing lipid bodies (LB), glycosomes (G), Golgi apparatus (GA), nucleus and kinetoplast (K), all well preserved (Fig. 2A). In contrast, parasites treated with butenafine EC50 presented external and internal morphological alterations. Butenafine-treated parasites did not present lesion in cell membrane (Fig. 2B and C), however parasites became morphologically round-shaped (Fig. 2C, inset). The cytoplasm presented a degenerated morphology, suggesting organelle degradation, the mitochondria (M) was fragmented, as observed in Fig. 2B, however kDNA presented normal morphology, blebs (B) containing parts of parasites were detected (Fig. 2C). Nuclear DNA seems to be fragmented (Fig. 2B; inset in 2C) and completely disorganized in some parasites, as observed in Fig. 2C. L. (L.) amazonensis promastigotes treated with $\mathrm{EC}_{50}$ of the standard drug, miltefosine, lost the fusiform morphology (Fig. 2D), the cytoplasm presented signs of degeneration with myelin-like structures (MS) and fragmented mitochondria (Fig. D). Nuclear DNA showed fragmented (Fig. D). Parasites treated with butenafine or miltefosine were also stained with Giemsa, and in this conditions, both butenafine - or miltefosine - treated $L$. (L.) amazonensis presented with round-shape morphology (data not shown), validating the external morphology observed in TEM images.

\subsection{Cytotoxicity and infection studies}

Butenafine and miltefosine induced mild cytotoxicity in peritoneal macrophages from BALB/c mice (data not show). In addition, butenafine and miltefosine presented similar $\mathrm{CC}_{50}$ that was estimated in $97.88 \pm$ 8.13 and $87.30 \pm 7.56 \mu \mathrm{M}$, respectively. 
Peritoneal macrophages infected with $L$. amazonensis or $L$. braziliensis were treated with 25,50 or $100 \mu \mathrm{M}$ of butenafine and with the estimated $\mathrm{EC}_{50}$ of miltefosine for L. amazonensis $(60 \mu \mathrm{M})$ and $L$. braziliensis $(64 \mu \mathrm{M})$ during $48 \mathrm{~h}$. The infection index of macrophages infected with $L$. amazonensis or L. braziliensis decreased in a dose-dependent manner, as showed in the Figs. $3 \mathrm{~A}$ and $\mathrm{B}$, respectively, and highlighted by images of macrophages infected with $L$. (L.) amazonensis (Fig. 3A, right side) or L. (V.) braziliensis (Fig. 3B right side) treated with butenafine or miltefosine. In addition, the concentration that decreased infection index to $50 \%$ was $29.81 \pm 7.34$ for L. amazonensis and $38.43 \pm$ $7.81 \mu \mathrm{M}$ for L. braziliensis.

\subsection{Nitric oxide and hydrogen peroxide}

The levels of $\mathrm{NO}$ and $\mathrm{H}_{2} \mathrm{O}_{2}$ in the supernatants of L. amazonensis- and L. braziliensis-infected macrophages treated or not with butenafine or miltefosine were quantified. The treatment with butenafine or with the $\mathrm{EC}_{50}$ of miltefosine did not change the levels of $\mathrm{NO}$ in macrophages infected with L. amazonensis (Fig. 4A) or L. braziliensis (Fig. 4B). Similarly, the levels of $\mathrm{H}_{2} \mathrm{O}_{2}$ were not changed in macrophages infected with $L$. amazonensis (Fig. 4C) or L. braziliensis (Fig. 4D) after the treatment with butenafine or miltefosine $\mathrm{EC}_{50}$.

\section{Discussion}

Considering that classic drugs used in the therapy for many infectious diseases, such as leishmaniasis, are considered highly toxic to patients, obsoletes as well as culminates in the emergence of drugresistance parasites, new prototype drugs need to be developed. In this regard, different methodologies have been used to characterize new active compounds, such as ethnopharmacology, chemotaxonomy and drug repositioning [23]. Drug repositioning is a rational option since the active compound is already approved for clinical use. Thus the new application for a known drug can result in a significant time and cost saving, being particularly important for neglected diseases. The use of already licensed products speed the way through pre-clinical efficacy tests aiming the use of these drugs [16]. For repositioning drugs in leishmaniasis it is necessary to understand 1) mechanism of action of the repurposed drug and 2) the biology of Leishmania parasites. It is well documented that during sterol biosynthesis Leishmania parasites produce ergosterol as one of final lipids of the cellular membrane. In this complex biochemical pathway, the enzyme squalene epoxidase is a key enzyme to produce lanosterol, the initial precursor of sterols. Thus, drugs such as butenafine that are able to specifically inhibit the action of this enzyme will impair physiological requirements of the parasites.

Indeed, promastigote forms of $L$. amazonensis and $L$. braziliensis treated with butenafine had their viabilities altered, being this drug at least two fold more effective than miltefosine for L. amazonensis, but for $L$. (V.) braziliensis butenafine was active in the same range as miltefosine, considering accuracy of their EC50s. In addition, this compound caused significant alterations in parasite ultrastructure. Parasites treated with $\mathrm{EC}_{50}$ of butenafine presented with cell shrinkage, intracellular disorganization, formation of blebs and fragmentation of nuclear DNA. Terbinafine, another allylamine compounds, was able to inhibit the multiplication of $L$. amazonensis promastigotes with $1 \mu \mathrm{M}$ and the main ultrastructural alterations was related to mitochondria impairment and also with formation of multivesicular bodies [33].

Although both terbinafine and butenafine compounds are able to inhibit squalene epoxidase, their leishmanicidal effects were different, as well as the possible pathway of death that drugs induced in $L$. amazonensis; these findings can have a direct correlation with the molecular structure of both compounds, that differs, because butenafine is a benzylamine, while terbinafine is an allylamine [30]. In fact, while terbinafine seems to interfere with lipid cycling in Leishmania sp., butenafine probably induces programmed cell death according to the morphological data. In parasites programmed cell death seems to be a predominant form of cell death in kinetoplastids in response to leishmanicidal compounds. In this regard, Arnoult et al. [3] showed by TEM that $L$. major promastigotes treated with staurosporine presented all morphological characteristics associated with apoptosis; Yamamoto et al. [36] found similar results after treatment of $L$. amazonensis with the triterpene ursolic acid. In addition, L. amazonensis incubated with miltefosine also presented ultrastructural characteristic of programmed cell death, such as cell shrinkage, fragmentation of mitochondria and nuclear DNA, and the presence of multivesicular structure. Previous studies also found similar morphological characteristic in L. donovani, L. major, L. tropica and L. amazonensis incubated with miltefosine [15,
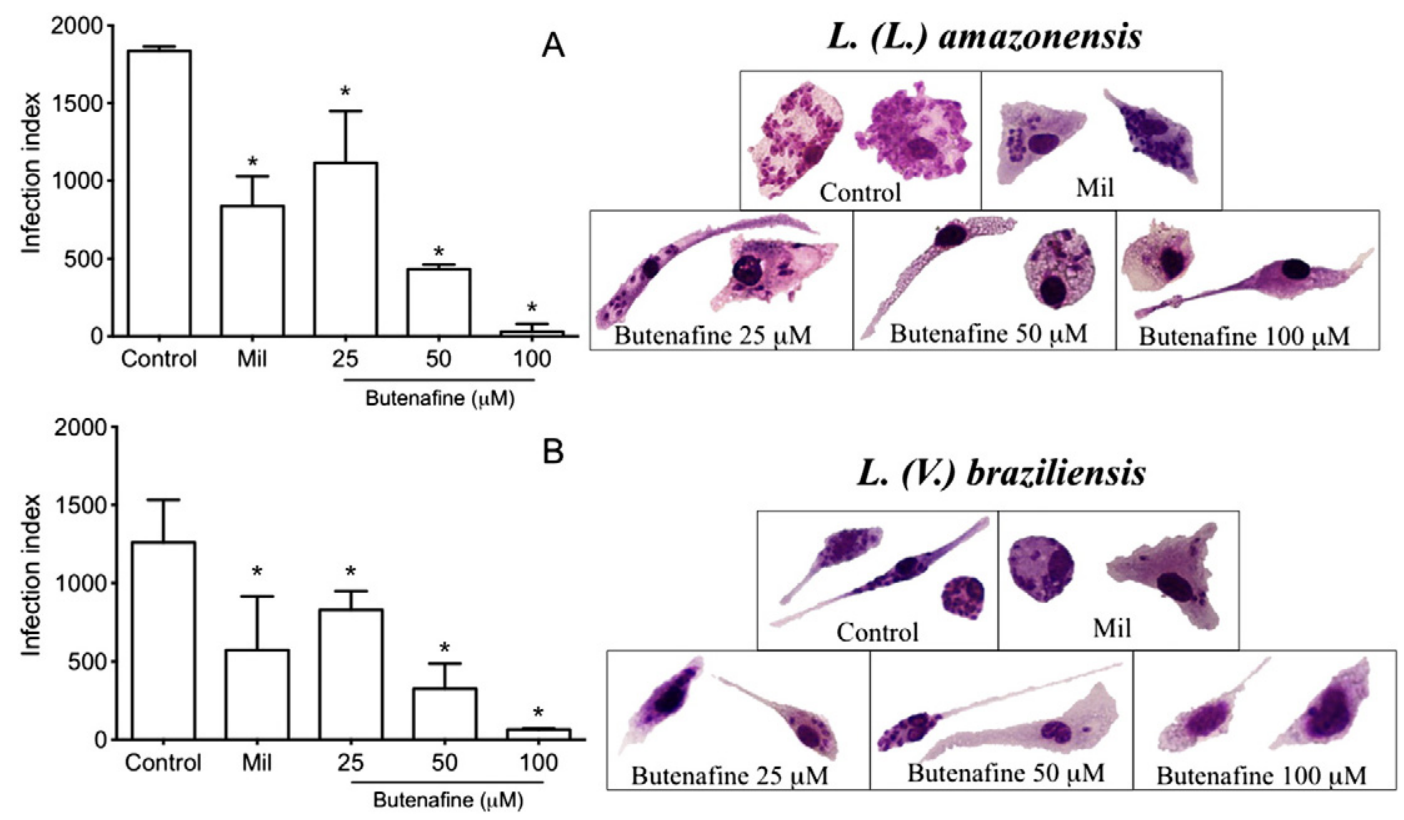

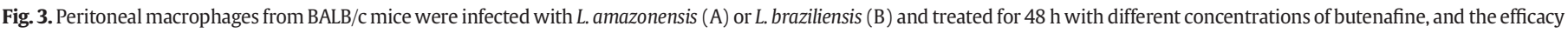

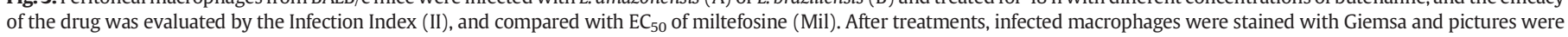
taken in order to demonstrate intracellular parasitism. ${ }^{*} \mathrm{p}<0.05$. 

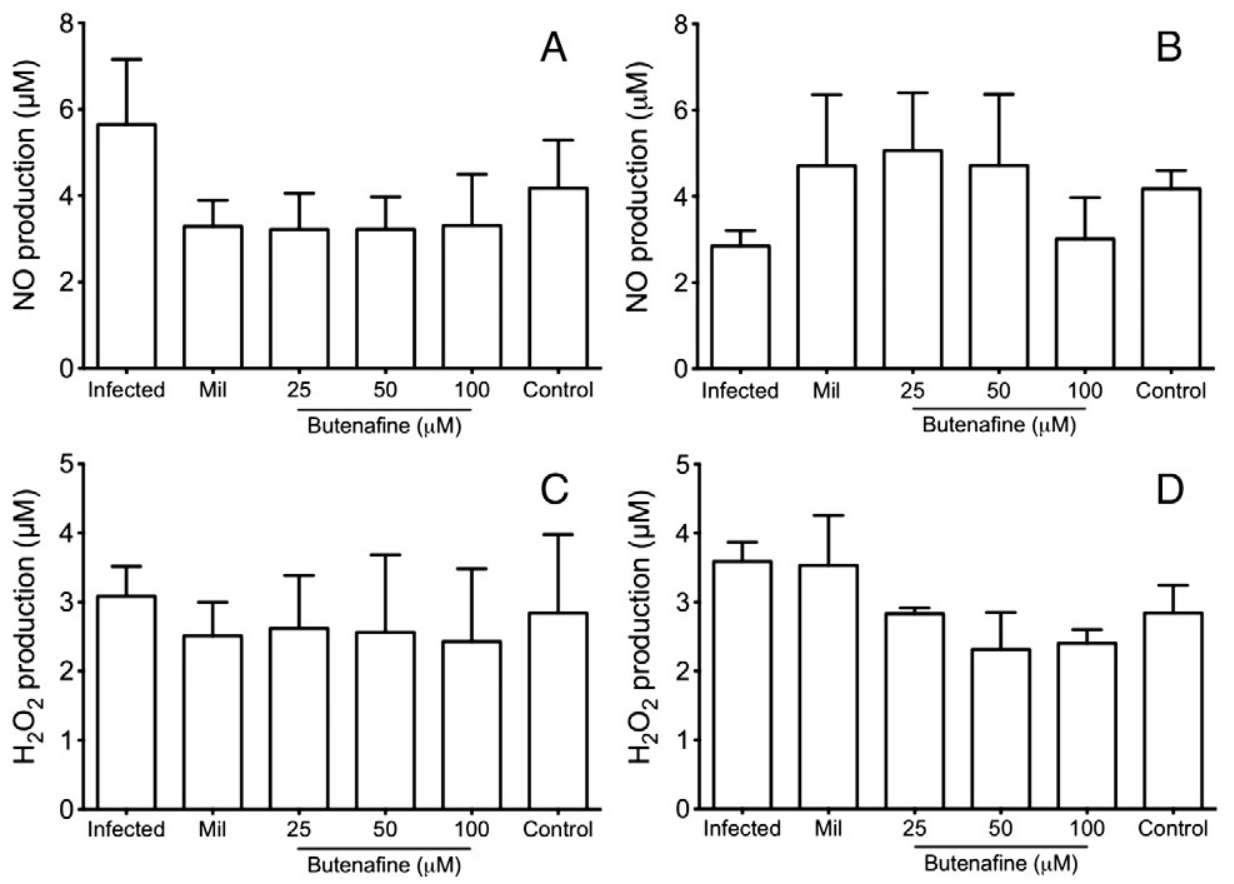

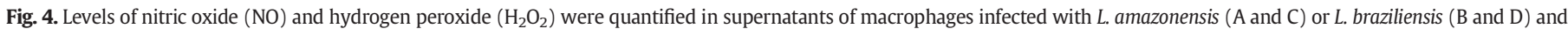
treated during $48 \mathrm{~h}$ with butenafine or miltefosine (Mil). ${ }^{*} \mathrm{p}<0.05$.

$19,21,34]$, that corroborate the results found with miltefosine in the present study. Thus, these data suggest that butenafine eliminated promastigote forms by a process of programmed cell death in $L$. amazonensis. Noteworthy, until now the effect of butenafine was not assayed in Leishmania parasites, and thus, description of its molecular target in this parasite genus is, indeed, unknown. In addition, differences in molecular structures of these compounds could have a direct impact in the molecular target of parasites, suggesting a different mechanism of death induced by allylamine and benzylamides.

In addition to the leishmanicidal effect of butenafine against the promastigote forms, in this study butenafine presented similar cytotoxicity than miltefosine in peritoneal macrophages, allowing its evaluation against the intracellular amastigotes. In this regard, it was verified that butenafine was able to eliminate amastigote forms of $L$. amazonensis and $L$. braziliensis, species that cause the most serious forms of cutaneous leishmaniasis in Latin America, the anergic diffuse and mucocutaneous leishmaniasis. Antifungal drugs such as ketoconazole, voriconazole, fluconazole, itraconazole and other azoles already were related to possess effect against amastigote forms of $L$. mexicana, $L$. amazonensis, $L$. major and $L$. donovani $[2,5,6,17,20]$. Similarly, the effect of antifungal allylamines was evaluated in leishmaniasis, and different studies showed that terbinafine, naftifine, and SF 86-327 drugs were able to eliminate intracellular or axenic amastigotes [6,33]. These results suggest that compounds capable of inhibiting enzymes responsible for ergosterol synthesis in Leishmania sp. will impact its viability. In fact, butenafine, a compound able to restrain the activity of squalene epoxidase enzyme, affected the viability of promastigotes and most importantly, intracellular amastigote forms of $L$. amazonensis and $L$. braziliensis, with high efficacy, being higher than miltefosine.

Some natural and synthetic compounds stimulate respiratory burst in macrophages, which in turn become competent to eliminate intracellular pathogens, such as Leishmania sp. [14,35]. In order to verify if the leishmanicidal activity of butenafine was mediated by $\mathrm{H}_{2} \mathrm{O}_{2}$ and $\mathrm{NO}$, the quantification of these reactive species were quantified. Butenafine did not alter the levels of $\mathrm{H}_{2} \mathrm{O}_{2}$ or $\mathrm{NO}$, suggesting a direct effect of butenafine on amastigote forms. Until now, studies have demonstrated that leishmanicidal effect of antifungal drugs, such as azoles or allylamines, are associated with a direct effect on parasites, targeting specifically the pathogen enzymes [9], and not stimulating the microbicidal effects as $\mathrm{H}_{2} \mathrm{O}_{2}$ or NO. The studies carried out with butenafine suggest that this drug eliminates directly the intracellular amastigotes of $L$. amazonensis and $L$. braziliensis destabilizing its morphology.

Taken together, the data presented herein suggest that butenafine eliminates promastigote forms by a mechanism associated with programmed cell death according to the morphological data, and in addition, this compound kill amastigote forms of $L$. amazonensis and $L$. braziliensis with superior efficacy than miltefosine. Although this is not the first study attesting leishmanicidal activity of antifungal compounds, it is the first one to demonstrate that butenafine shows leishmanicidal activity, adding valuable information to the countable chemotherapeutical arsenal of leishmaniasis.

\section{Acknowledgment}

The authors would like to thank the FAPESP (2015/18746-4) and HCFMUSP-LIM50.

\section{References}

[1] K. Aït-Oudhia, E. Gazanion, D. Sereno, B. Oury, J.P. Dedet, F. Pratlong, L. Lachaud, In vitro susceptibility to antimonials and amphotericin B of Leishmania infantum strains isolated from dogs in a region lacking drug selection pressure, Vet. Parasitol. 187 (2012) 386-393.

[2] V.V. Andrade-Neto, T.M. Pereira, M. Canto-Cavalheiro, E.C. Torres-Santos, Imipramine alters the sterol profile in Leishmania amazonensis and increases its sensitivity to miconazole, Parasit Vectors 9 (2016) 183.

[3] D. Arnoult, K. Akarid, A. Grodet, P.X. Petit, J. Estaquier, J.C. Ameisen, On the evolution of programmed cell death: apoptosis of the unicellular eukaryote Leishmania major involves cysteine proteinase activation and mitochondrion permeabilization, Cell Death Differ. 9 (2002) 65-81.

[4] J.D. Berman, J.G.G. Holz, D.H. Beach, Effects of ketoconazole on growth and sterol biosynthesis of Leishmania mexicana promastigotes in culture, Mol. Biochem. Parasitol. 12 (1984) 1-13.

[5] J.D. Berman, L.J. Goad, D.H. Beach, J.G.G. Holz, Effects of ketoconazole on sterol biosynthesis by Leishmania mexicana mexicana amastigotes in murine macrophage tumor cells, Mol. Biochem. Parasitol. 20 (1986) 85-92.

[6] J.D. Berman, J.V. Gallalee, In vitro antileishmanial activity of inhibitors of steroid biosynthesis and combinations of antileishmanial agents, J. Parasitol. 73 (1987) 671-673. 
[7] A. Chattopadhyay, M. Jafurulla, A novel mechanism for an old drug: amphotericin $B$ in the treatment of visceral leishmaniasis, Biochem. Biophys. Res. Commun. 416 (2011) 7-12.

[8] S. S. Das, J.N. Barbhuniya, I. Biswas, S. Bhattacharya, P.K. Kundu, Studies on comparison of the efficacy of terbinafine $1 \%$ cream and butenafine $1 \%$ cream for the treatment of Tinea cruris, Indian Dermatol Online J. 1 (2010) 8-9.

[9] T.P.C. Dorlo, M. Balasegaram, J.H. Beijnen, P.J.V. de, Miltefosine: a review of its pharmacology and therapeutic efficacy in the treatment of leishmaniasis, J. Antimicrob. Chemother. 67 (2012) 2576-2597.

[10] T.P.C. Dorlo, T.A. Eggelte, P.J.V. de, J.H. Beijnen, Characterization and identification of suspected counterfeit miltefosine capsules, Analyst 137 (2012) 1265-12SU74.

[11] E.K. Elmahallawy, A. Agil, Treatment of leishmaniasis: a review and assessment of recent research, Clin. Infect. Dis. 60 (2015) 1398-1404.

[12] L.H. Freitas-Junior, E. Chatelain, H.A. Kim, J.L. Siqueira-Neto, Visceral leishmaniasis treatment: What do we have, what do we need and how to deliver it? Int. J. Parasitol. Drugs Drug Resist. 2 (2012) 11-19.

[13] L.J. Goad, R.L. Berens, J.J. Marr, D.H. Beach, J.G.G. Holz, The activity of ketoconazole and other azoles against Trypanosoma cruzi: biochemistry and chemotherapeutic action in vitro, Mol. Biochem. Parasitol. 32 (1989) 179-189.

[14] C. Hernández-Chinea, E. Carbajo, F. Sojo, F. Arvelo, V.V. Kouznetsov, A.R. RomeroBohórquez, P.J. Romero, In vitro activity of synthetic tetrahydroindeno[2,1-c]quinolines on Leishmania Mexicana, Parasitol. Int. 64 (2015) 479-483.

[15] S. Khademvatan, M.J. Gharavi, F. Rahim, J. Saki, Miltefosine-induced apoptotic cell death on Leishmania major and L. tropica strains, Korean J. Parasitol. 49 (2011) $17-23$.

[16] D.M. Klug, M.H. Gelb, M.P. Pollastri, Repurposing strategies for tropical disease drug discovery, Bioorg. Med. Chem. Lett. 26 (2016) 2569-2576.

[17] M.M. Kulkarni, N. Reddy, T. Gude, B.S. McGwire, Voriconazole suppresses the growth of Leishmania species in vitro, Parasitol. Res. 112 (2013) 2095-2099.

[18] L.V.R. Lima, P.K.R. Santos, M.B. Campos, T.V. dos Santos, C.M. de Castro Gomes, M.D. Laurenti, C.E. Corbett, F.T. Silveira, Preclinical diagnosis of American visceral leishmaniasis during early onset of human Leishmania (L.) infantum chagasi-infection, Pathog. Glob. Health 108 (2014) 381-384.

[19] A. Fde, K.C. Marinho, S.S. Gonçalves, A.C. Oliveira, M. Oliveira, C.M.d.'A.-L. Bellio, A.L. Santos, M.H. Branquinha, Miltefosine induces programmed cell death in Leishmania amazonensis promastigotes, Mem. Inst. Oswaldo Cruz 106 (2011) 507-509.

[20] T. Pandharkar, X. Zhu, R. Mathur, J. Jiang, T.D. Schmittgen, C. Shaha, K.A. Werbovetz, Studies on the antileishmanial mechanism of action of the arylimidamide DB766: azole interactions and role of CYP5122A1, Antimicrob. Agents Chemother. 58 (2014) 4682-4689.

[21] C. Paris, P.M. Loiseau, C. Bories, J. Bréard, Miltefosine induces apoptosis-like death in Leishmania donovani promastigotes, Antimicrob. Agents Chemother. 48 (2004) 852-859.

[22] L.F. Passero, J.V. Sacomori, T.Y. Tomokane, C.E. Corbett, F.T. da Silveira, M.D. Laurenti, Ex vivo and in vivo biological behavior of Leishmania (Viannia) shawi, Parasitol. Res. 105 (6) (2009 Nov) 1741-1747.

[23] L.F. Passero, M.D. Laurenti, G. Santos-Gomes, B.L. Soares Campos, P. Sartorelli, J.H. Lago, Plants used in traditional medicine: extracts and secondary metabolites exhibiting antileishmanial activity, Curr. Clin. Pharmacol. 9 (2014) 187-204.
[24] M. Perry, S. Wyllie, V. Prajapati, J. Menten, A. Raab, J. Feldmann, D. Chakraborti, S. Sundar, M. Boelaert, A. Picado, A. Fairlamb, Arsenic, antimony, and Leishmania: has arsenic contamination of drinking water in India led to treatment-resistant kalaazar? Lancet 385 (2015) S80.

[25] B. Purkait, R. Singh, K. Wasnik, S. Das, A. Kumar, M. Paine, M. Dikhit, D. Singh, A.H. Sardar, A.K. Ghosh, P. Das, Up-regulation of silent information regulator 2 (Sir2) is associated with amphotericin B resistance in clinical isolates of Leishmania donovani, J. Antimicrob. Chemother. 70 (2015) 1343-1356.

[26] H. Rangel, F. Dagger, A. Hernandez, A. Liendo, J.A. Urbina, Naturally azole-resistant Leishmania braziliensis promastigotes are rendered susceptible in the presence of terbinafine: comparative study with azole-susceptible Leishmania mexicana promastigotes, Antimicrob. Agents Chemother. 40 (1996) 2785-2791.

[27] R.N. Sampaio, G.H. Takano, A.C. Malacarne, T.R. Pereira, A.V. de Magalhães, In vivo Terbinafine inefficacy on cutaneous leishmaniasis caused by Leishmania (Leishmania) amazonensis in C57BL/6 mice, Rev. Soc. Bras. Med. Trop. 36 (2003) 531-533.

[28] F.T. Silveira, R. Lainson, C.M. De Castro Gomes, M.D. Laurenti, C.E. Corbett, Immunopathogenic competences of Leishmania $(V$.) braziliensis and $L$. (L.) amazonensis in American cutaneous leishmaniasis, Parasite Immunol. 31 (2009) 423-431.

[29] L. Simões-Mattos, M.J. Teixeira, D.C. Costa, J.J.R. Prata, C.M. Bevilaqua, J.J. Sidrim, M.F. Rocha, Evaluation of terbinafine treatment in Leishmania chagasi-infected hamsters (Mesocricetus auratus), Vet. Parasitol. 103 (2002) 207-216.

[30] D. Sudip, J.N. Barbhuniya, S.I. Biswas, S. Bhattacharya, P.K. Kundu, Studies on comparison of the efficacy of terbinafine $1 \%$ cream and butenafine $1 \%$ cream for the treatment of Tinea cruris, Indian Dermatol. Online J. 1 (2010) 8-9.

[31] S. Sundar, J. Chakravarty, An update on pharmacotherapy for leishmaniasis, Expert. Opin. Pharmacother. 16 (2015) 237-252.

[32] C.P. Thakur, R.K. Singh, S.M. Hassan, R. Kumar, S. Narain, A. Kumar, Amphotericin B deoxycholate treatment of visceral leishmaniasis with newer modes of administration and precautions: a study of 938 cases, Trans. R. Soc. Trop. Med. Hyg. 93 (1999) 319-323.

[33] M.A. Vannier-Santos, J.A. Urbina, A. Martiny, A. Neves, W. de Souza, Alterations induced by the antifungal compounds ketoconazole and terbinafine in Leishmania, J. Eukaryot. Microbiol. 42 (1995) 337-346.

[34] N.K. Verma, C.S. Dey, Possible mechanism of miltefosine-mediated death of Leishmania donovani, Antimicrob. Agents Chemother. 48 (2004) 3010-3015.

[35] E.S. Yamamoto, B.L. Campos, M.D. Laurenti, J.H. Lago, S. Sdos, C.E.C. Grecco, L.F.D. Passero, Treatment with triterpenic fraction purified from Baccharis uncinella leaves inhibits Leishmania (Leishmania) amazonensis spreading and improves Th1 immune response in infected mice, Parasitol. Res. 113 (2014) 333-339.

[36] E.S. Yamamoto, B.L. Campos, J.A. Jesus, M.D. Laurenti, S.P. Ribeiro, E.G. Kallá, M. Rafael-Fernandes, G. Santos-Gomes, M.D. Silva, D.P. Sessa, J.H. Lago, D. Levy, L.F.D, Passero, The effect of ursolic acid on Leishmania (Leishmania) amazonensis is related to programed cell death and presents therapeutic potential in experimental cutaneous leishmaniasis, PLoS One 10 (2015) e0144946. 